\title{
Peripheral and foveal segmentation of angle textures
}

\author{
CRISTINA MEINECKE \\ University of Munich, Munich, Germany \\ and \\ LOTHAR KEHRER \\ University of Bielefeld, Bielefeld, Germany
}

\begin{abstract}
Studies of the effects of retinal eccentricity on the visual segmentation of textures are presented. The textures used in these studies were composed of angle elements. These were presented tachistoscopically to college students in three different experiments. Results showed that there were different relationships between segmentation performance and eccentricity, depending on the width of the angles used in the background and target texture. One major difference was that peak performance was found in the fovea in some conditions, and in peripheral areas in other conditions. Performance in the fovea and the periphery seemed to be determined by qualitatively different features. It was assumed that an appropriate explanation is that the system-internal representation of a specific stimulus within the early visual system differs as a function of the retinal location at which it is projected. Thus, the critical features discriminating between target and background texture have to be sought in the system-internal representation of the stimulus instead of in the stimulus itself. The data show that a relatively exact system-internal representation of the stimulus is present in the fovea, where performance is determined by angle width. In the periphery, in contrast, angles seem to be represented as "blobs," and performance is determined by the orientation of the blobs' main axes.
\end{abstract}

One of the issues studied in research on preattentive visual segmentation is which stimulus features lead to a segmentation of the stimulus into various areas. There is much evidence that segmentation can be produced by differences in color (e.g., Carter, 1982; Farmer \& Taylor, 1980; McIlhagga, Hine, Cole, \& Snyder, 1990), luminance (e.g., Beck, Graham, \& Sutter, 1991; Deubel, Findlay, Jacobs, \& Brogan, 1988; Engel, 1974; Nothdurft, 1985b), and texture (e.g., Beck, 1982; Bergen, 1991; Caelli, 1985; Fox \& Mayhew, 1979; Graham, 1989; Nothdurft, 1990). However, in textures, it is not always possible to identify the specific stimulus properties that are relevant for performance. An example of such a texture can be found in Olson and Attneave (1970). They constructed several textures consisting of different arrangements of right angles in the target and background areas and compared perceptual segmentation performance in two stimulus patterns, as shown in Figure 1. Their data showed that the target area can be segmented with relatively little effort in the stimulus pattern shown in Fig-

\footnotetext{
We wish to thank Philip J. Kellman for his helpful suggestions, Werner Schubö for statistical support, and Jonathan Harrow (Max Planck Institute for Psychological Research, Munich) for translating this text from the German. We wish to thank the German Research Association (DFG) for their financial support in carrying out these studies. Address correspondence to C. Meinecke, Institut für Psychologie, Universität München, Leopoldstr. 13, D-80802 München, Germany.
}

ure 1a, but not in the pattern in Figure 1b. Beck (1972) has also reported similar findings. How does this difference in segmentation performance arise?

A closer inspection of the stimulus patterns reveals that this difference cannot be due to the lines from which each angle is constructed, because in the two conditions they exhibit the same $\left(45^{\circ}\right)$ orientations in both target and background. It could be assumed that texture segmentation is caused by the difference in the orientation of the angle as a whole. A series of studies has shown that orientation difference is a relevant feature in texture segmentation performance (e.g., Beck, 1966; Bergen \& Julesz, 1983; Caelli \& Julesz, 1979; Deubel \& Frank, 1991; Nothdurft, 1991; Sagi \& Julesz, 1987). Nonetheless, how can the orientation of an angle be defined? Basically, there are several possibilities-for example, the direction in which the apex of an angle points, or the direction of the side that would transform the angle into a triangle. However, the literature suggests that the direction of the greatest extent of an angle defines its orientation (see Beck, 1972; Olson \& Attneave, 1970). This is a promising idea that we have taken up and subjected to a (previously unperformed) test.

Now, how can the extent of an angle be operationalized? Fox and Mayhew (1979) have proposed the following procedure. With reference to Marr's (1976) ideas on the primal sketch, they assume that, in early vision, each angle is represented in the visual system as a "blob." 
For the sake of simplicity, these blobs can be imagined as ellipses in which the angles are embedded. The orientation of an angle is then defined by the direction of the major axis of the ellipse.

Figure 2 presents the relation between the width of the angle and the orientation of the blob for isosceles angles. Angles with a width of less than $60^{\circ}$ are represented as blobs oriented in the direction of the bisector of the angle; angles with a width of $60^{\circ}$ produce blobs with no specific orientation as the ellipse forms a circle; and angles of more than $60^{\circ}$ produce blobs with an orientation in a direction that is the same as that for the side that would transform the angle into a triangle.

This operationalization of angle orientation could provide a good explanation for the differences in the perceptual segmentation of the two stimulus patterns in Figure $1\left(90^{\circ}\right.$ angle, differently arranged). In the stimulus pattern shown in Figure 1a, the angles in the target and background areas have different orientations. The angles represented as blobs are perpendicular to each other. In Figure $1 \mathrm{~b}$, in contrast, the angles in both target and background areas show the same (horizontal) orientation.

In the present study we describe three experiments that were all designed according to the same logic: Subjects have to segment a target area from the background. In one condition, angles whose blobs revealed no differences in orientation were used in both the background and the target area; both angles were either larger or smaller than $60^{\circ}$. We labeled this condition same. In a second condition, we used angles in the target area whose blobs showed an orientation that was different from that of the blobs of the angles in the background; one angle was smaller than $60^{\circ}$, and the other was larger. We labeled this condition different.

If angles are represented as blobs in early vision, and if differences in the orientation of these blobs can be exploited, performance should be better in the different condition than in the same condition, because in the former, the blobs in the target area and the background have different orientations. This hypothesis is based on the angles' being represented as blobs, so we have called

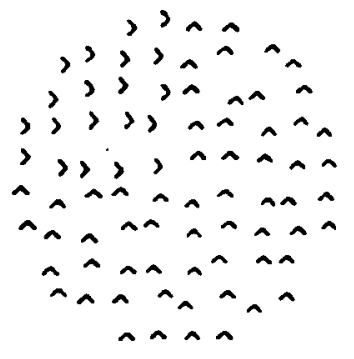

a)

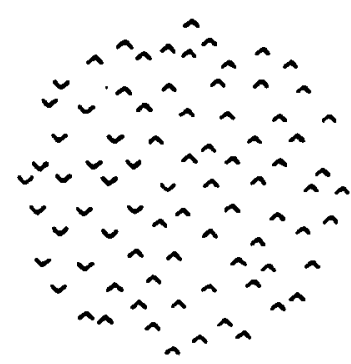

b)
Figure 1. Textures with $90^{\circ}$ angles. Angles in the target area are rotated by $90^{\circ}$ (a) and $180^{\circ}$ (b). Adapted from "What Variables Produce Similarity Grouping?" by R. K. Olson and F. Attneave, 1970, American Journal of Psychology, 83, p. 5. Copyright 1970 by the University of Illinois Press. Adapted by permission. (Note that Cat. 9 in the original has been rotated to bring about Figure 1a.)

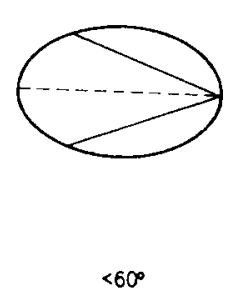

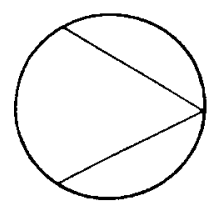

$60^{\circ}$

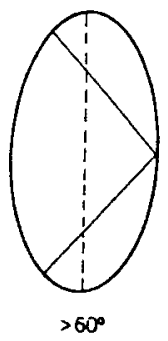

Figure 2. Three angles with different widths and their surrounding ellipses with their major axes. The ellipses are constructed in such a way that they touch the comers of the (imaginary) triangle, and the center of gravity of the triangle is simultaneously the center of the ellipses.

angles' being represented as blobs, so we have called this the blob hypothesis.

\section{EXPERIMENT 1}

In both conditions of Experiment 1, the background consisted of angles with a width of $50^{\circ}$ (see Figure 3 ). In the same condition, target angles had widths of $30^{\circ}$. This made both angles smaller than $60^{\circ}$, and both target and background blobs did not exhibit any differences in the orientation of their major axes-they were horizontal. In the different condition, target angles had widths of $70^{\circ}$, and thus the target blobs had an orientation that was different from that of the background blobs-they were vertical.

All other differences between target and background elements were the same in both conditions. The width of the target and background angles differed by $20^{\circ}$, the apexes of target and background angles faced in opposite directions, and the imaginary sides that would transform the angle into a triangle always had a vertical orientation.

The subjects' task was to detect the target. Other studies have shown that detection performance in segmentation tasks is not necessarily optimal in the fovea (see Kehrer, 1987, 1989), so the retinal eccentricity of the target projection was varied in this experiment.

\section{Method}

Subjects. Eight female subjects and 1 male subject were paid to participate in the experiment. All the subjects reported normal or fully corrected visual acuity.

Apparatus. The stimuli were presented on a Hewlett Packard 1311B X-Y display (167-Hz refresh rate in our experiment) connected to an HP graphics generator (1351 A). The graphics generator was under the control of an Atari microcomputer (1040 STF). The display was illuminated with two incandescent lamps in such a way that no reflections were visible on the surface of the screen. These lighting conditions resulted in a luminance of 1.7 $\mathrm{cd} / \mathrm{m}^{2}$ for the background. In order to obtain the luminance for the stimulus lines, these were placed together without any space between them, and then a value of $84 \mathrm{~cd} / \mathrm{m}^{2}$ was measured across several lines.

The subject sat at a table on which a head- and chinrest was mounted. The display monitor was positioned to give an observation distance of $50 \mathrm{~cm}$ with the direction of gaze inclined slightly 

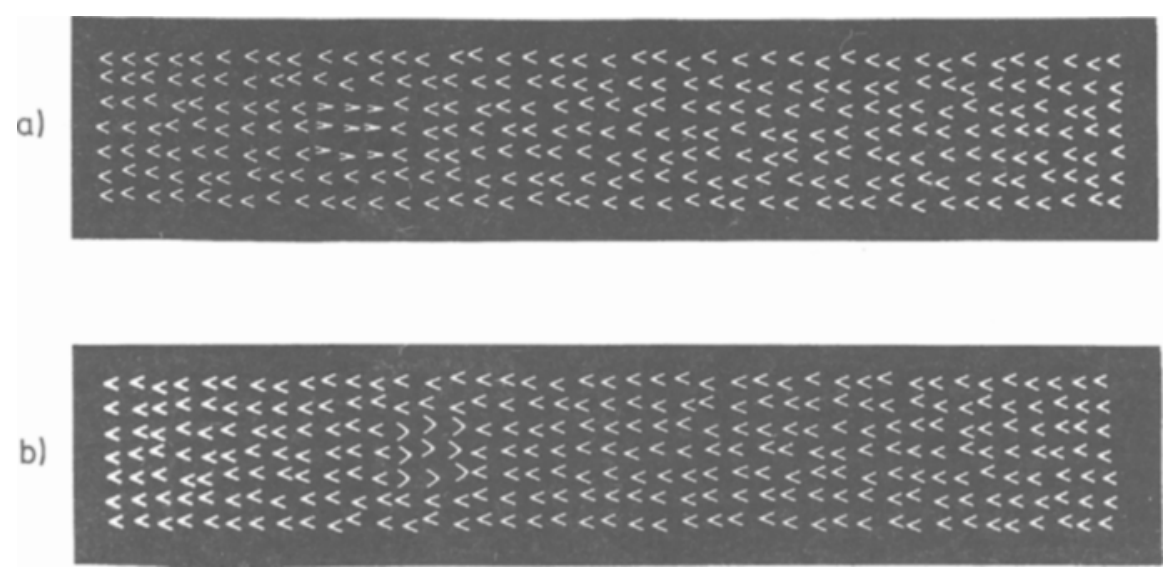

Figure 3. Stimulus patterns of Experiment 1. Angle width target/background: (a) same condition, $30^{\circ} / 50^{\circ}$; (b) different condition, $70^{\circ} / 50^{\circ}$.

downward (approximately $5^{\circ}$ ). Two response keys set on a single box were placed on the table. The subject was able to move the response keys, and they could be comfortably operated with the index fingers of both the left and right hands.

Stimuli. The stimulus display consisted of 287 angles: 41 horizontal $\times 7$ vertical. The repeat intervals were varied randomly within set margins (no neighboring angles touched or crossed) in order to offset the increase in local luminance that occurs in an equidistant arrangement of lines (see Kehrer, 1987; Nothdurft, 1985a). The stimulus field subtended $24^{\circ}$ in the horizontal direction and $3.1^{\circ}$ in the vertical direction. The size of the target texture was $1.4^{\circ}$ in both the vertical and the horizontal directions.

The subject had to detect a target texture that could be found $(p=.5)$ within a background texture (the context). The context was composed of angles with their bisectors on an imaginary horizontal line. The value of their width varied according to the particular experimental condition. In the stimuli containing a target texture, a patch of $3 \times 3$ background angles was replaced by target angles with different widths. In addition, the target angles were tilted at $180^{\circ}$ to the background angles.

The vertical position of the target texture was held constant exactly in the middle of the context. The horizontal position of the target patch was varied. In addition, an area of two angles was left uncovered by the target texture on both the left and right edges of the stimulus. This resulted in a total of 35 possible positions for the target texture.

Procedure. In each session, the subjects were confronted with a total of 560 stimulus presentations separated into four blocks of 140 trials with a short break between each block. The total of 140 trials in each block was made up of two presentations of the target texture in each of the 35 possible positions, combined with 70 stimuli that did not contain a target (i.e., negative trials). The sequence of positive and negative trials and the position of the target in each positive trial was randomized. Thus, the subjects could not predict whether or not the next trial would contain a target, and if it contained one, they did not know in which position it would appear. The stimuli were presented for $80 \mathrm{msec}$.

In two of the four blocks in each session, the stimulus display consisted of context angles with widths of $50^{\circ}$ and target angles with widths of $30^{\circ}\left(30^{\circ} / 50^{\circ}\right.$; same condition). In the remaining two blocks, the target angles showed widths of $70^{\circ}$; the context angles were held constant at $50^{\circ}\left(70^{\circ} / 50^{\circ}\right.$; different condition). The sequence of the two conditions was alternated and balanced across subjects and was changed for each subject from one session to the next. Four sessions were administered on each of 4 successive days. Each session took about $45 \mathrm{~min}$.
Each stimulus presentation was preceded by a "colon" figure that was displayed in the middle of the monitor (two vertically oriented pixels with a distance of $2 \cdot 1^{\circ}$ ). This informed the subject that the computer was ready, and that he or she could summon the first or next stimulus display. The subject could initiate the stimulus presentation by simultaneously pressing both response buttons. The computer then replaced the colon with a fixation point in the middle of the monitor. This was automatically replaced by the stimulus after a period of $800 \mathrm{msec}$. The stimulus was replaced by a masking stimulus after $80 \mathrm{msec}$. The mask consisted of superimposed lines with an orientation that was the same as that for the lines forming the context angles. A fixation point one pixel in size was preferred to a fixation cross in order to keep forward masking effects as small as possible (see Kehrer, 1987).

The mask remained on the monitor until the subject responded by pressing either the left or right button on the response box. In the instructions, the subjects were told to press the right button for positive reactions (target present) and the left button for negative reactions (no target present). If the subject's response was correct, the keypress was followed by the appearance of the "ready" colon, indicating that a new trial could be initiated. When an incorrect response was made, the previous stimulus configuration was repeated for a period of $1.5 \mathrm{sec}$ to provide feedback. This repetition was followed by the colon, as for a correct response.

The subjects were instructed to fixate the fixation point as closely as possible, to respond as quickly as possible, and to make as few false alarms as possible. The last point was introduced to avoid incorrect "yes" responses (false alarms), so that a subject would only give a "yes" response if he or she was relatively certain that the stimulus contained a target. The purpose of this instruction was to keep interindividual criterion differences as low as possible.

\section{Results}

Figure 4 presents the percentage of hits as a function of the eccentricity of the target. These data were subjected to an arcsine transformation (to stabilize the variance; Sachs, 1978) and subsequently used to compute an analysis of variance (ANOVA) for repeated measures with the variables condition (same vs. different), position (1-35), and session $(2,3,4)$. In the different condition, targets were detected significantly more frequently than they were in the same condition $[F(1,8)=11.42$, $p<.01]$. There was a significant effect of position 


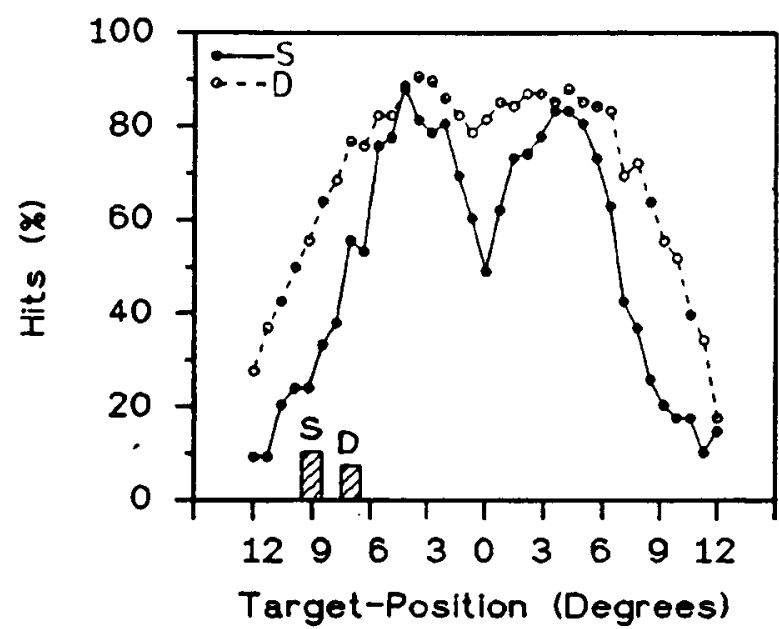

Figure 4. Percentage of hits as a function of retinal eccentricity (degrees) in the same condition (S) and the different condition (D) in Experiment 1 . Blocks show false alarms.

$[F(34,272)=27.64, p<.001]$ and session $[F(2,16)=$ $11.64, p<.001]$. There was also a significant interaction between condition and position $[F(34,272)=1.87, p<$ $.01]$. All other interactions were nonsignificant.

\section{Discussion}

The results show that, in line with the blob hypothesis, detection performance in the different condition was better than that in the same condition. This supports the idea that angles are represented as blobs in early vision, and that the orientation of these blobs is exploited during segmentation of the stimulus pattern.

In addition, the results confirm Kehrer's $(1987,1989)$ finding that maximum segmentation performance is not always found at the fovea, but can be found in more parafoveal areas as well. This performance drop at the foveal center will be discussed more thoroughly in the General Discussion.

Thus, we can initially state that Experiment 1 provides evidence in support of the blob hypothesis. However, against this background, one secondary finding needs to be explained. If the orientation differences of blobs cannot be exploited in the same condition, we have to ask why detection performance in this condition was not generally much worse. There seem to be eccentricities at which there are no essential differences in performance under these two conditions (at approximately $3^{\circ}$ ). So, it may be that differences in the orientation of blobs as well as other features of the stimulus are relevant for segmentation. We attempted to gain more information on this hypothesis in Experiment 2.

\section{EXPERIMENT 2}

In Experiment 1, we varied the orientation of blobs by constructing angles of varying widths. In the same condition, subjects had to detect small angles $\left(30^{\circ}\right)$ among large angles $\left(50^{\circ}\right)$. In the different condition, large angles $\left(70^{\circ}\right)$ had to be detected among small angles $\left(50^{\circ}\right)$. It is well known that "size" is a discriminatory feature that can influence texture segmentation (Bergen \& Adelson, 1988; Cavanagh, Arguin, \& Treisman, 1990; Engel, 1974; Gurnsey \& Browse, 1987; Treisman \& Gelade, 1980). Therefore, it cannot be ruled out that differences in size between target and background angles might have determined segmentation performance in Experiment 1.

In addition, texture segmentation experiments with so-called asymmetric stimulus conditions have shown that large texture elements are easier to detect within small background elements than are small target elements within large background elements ("More in less is better than less in more"; Gurnsey \& Browse, 1989 , p. 37). This finding could even explain the superiority of the different condition $\left(70^{\circ} / 50^{\circ}\right)$ over the same condition $\left(30^{\circ} / 50^{\circ}\right)$ in the present Experiment 1.

In summary, the difference in performance between the two conditions in Experiment 1 could also be attributed to the asymmetrical distribution of the feature "size" between target and background elements. We call this the asymmetry hypothesis.

Experiment 2 was designed to test the contributions of the blob hypothesis and the asymmetry hypothesis to explaining the difference in performance between the same and different conditions. Design and procedure were exactly the same as those in Experiment 1. Once more, there was a different condition, in which the target and background blobs differed in orientation. In the same condition, the target and background blobs did not differ in orientation. The background consisted of angles with widths of $70^{\circ}$. In the different condition, the target angle had a width of $50^{\circ}$; in the same condition, it had a width of $90^{\circ}$ (see Figure 5).

According to the blob hypothesis, detection performance should be better in the different condition $\left(50^{\circ} / 70^{\circ}\right)$, because differences in orientation could be exploited there. According to the asymmetry hypothesis, detection performance should be better in the same condition $\left(90^{\circ} / 70^{\circ}\right)$, because "large" target elements had to be detected within "small" background elements there.

\section{Method}

Subjects. Six female and 4 male subjects were paid to participate in the experiment. All the subjects reported normal or fully corrected visual acuity.

Apparatus and Procedure. These did not differ from those of Experiment 1.

Stimuli. Angles were constructed in line with the ideas presented in the introduction.

\section{Results}

Figure 6 presents the percentage of hits as a function of the eccentricity of the target. It shows a clear crossover of the two curves at an eccentricity of approximately $3.5^{\circ}$. As in Experiment 1, the data were subjected to an arcsine transformation and subsequently used to compute an ANOVA for repeated measures with the variables of condition (same vs. different), position (1-35), and session $(2,3,4)$. The difference between the two 
a)
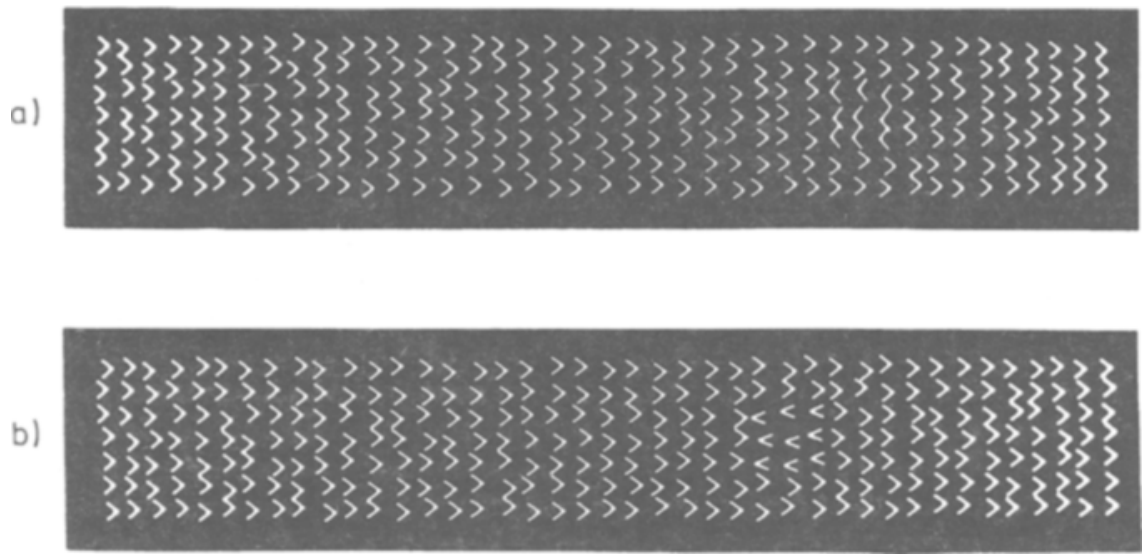

Figure 5. Stimulus patterns of Experiment 2. Angle width target/background: (a) same condition, $90^{\circ} / 70^{\circ}$; (b) different condition, $50^{\circ} / 70^{\circ}$.

conditions just failed to reach significance $[F(1,9)=$ $4.96, p=.053]$. There was a significant effect of position $[F(34,306)=14.62, p<.001]$ and session $[F(2,18)=$ $12.34, p<.001]$. There was also a significant interaction between condition and position $[F(34,306)=12.24, p<$ $.001]$. All other interactions were nonsignificant.

Orthogonal polynomial contrasts were calculated for the variable position. The results showed that the quadratic and the quartic effects of the variable position were different for both conditions [quadratic interaction condition/position, $F(1,9)=67.20, p<.001$; quartic, $F(1,9)=52.50, p<.001]$. This result indicates that the subtraction of the two conditions yields a value with a sign in the central area that is different from that in the two peripheral areas.

\section{Discussion}

The analysis of the data has shown that neither the blob nor the asymmetry hypothesis can (or should) be

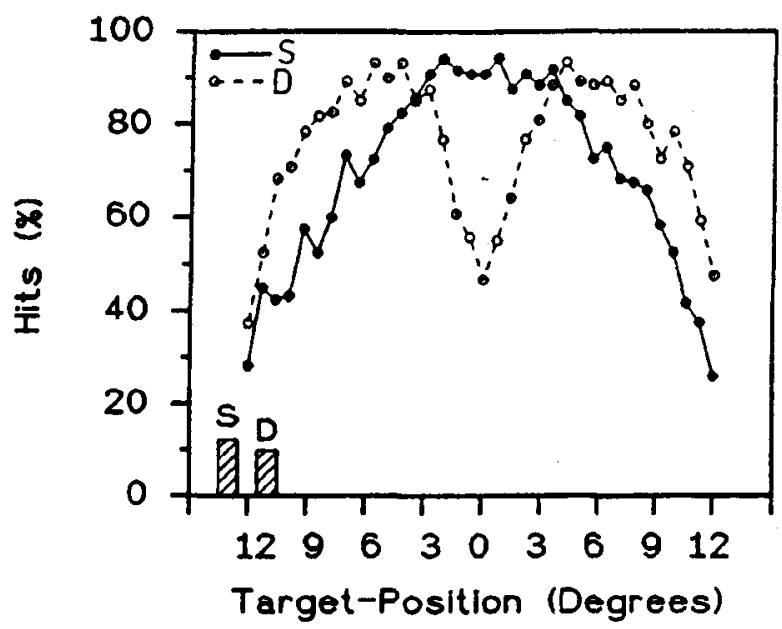

Figure 6. Percentage of hits as a function of retinal eccentricity (degrees) in the same condition (S) and the different condition (D) in Experiment 2. Blocks show false alarms. rejected. It is far more the case that, depending on retinal eccentricity, either of the hypotheses provides a good explanation of the findings. The fovea shows a clear performance advantage when large angles have to be detected within small ones. In the fovea, the pattern of findings predicted by the asymmetry hypothesis was obtained. In contrast, the periphery is completely different in that it seems that the early visual system exploits differences in the orientations of blobs there. Thus, the findings suggest that the blob hypothesis should be retained as an approach to explaining detection performance in angle textures, although its area of impact should be restricted to the periphery.

A slight restriction has to be made concerning the critical feature, which determines the performance in the foveal area. In Figure 5a (same condition), the large angles in the target region seem to touch each other in some cases. This leads to the impression of longer line segments in the target than in the context region. Therefore, one cannot exclude that good performance in the foveal region is determined not only by the aperture differences of the angles, but also by the feature of "line length." This does not concern performance in the periphery, where performance in the same condition was poorer than that in the different condition; here, this additional feature of "line length" did not help the subjects find the target.

The results of Experiment 1 can be reconsidered against the background of Experiment 2. There is some support for attributing the general performance advantage in the different condition compared with the same condition (see Figure 4) to two causes. (1) In the periphery, better detection performance results from differences in the orientation of blobs. (2) In the fovea, in contrast, better detection performance is due to favorable asymmetrical relationships between the sizes of the angles (or to some other feature).

This experiment also reveals a very strong performance drop in the fovea in one condition. This finding will be considered in more detail in the General Discus- 
sion, but first we present a third experiment, which was needed to strengthen the findings in Experiment 2. In the prior experiments, the apexes of target and background angles pointed in opposite directions. As mentioned in the introduction, the direction of the angle apexes may also represent an operationalization of angle orientation. Although this discriminatory feature was found in both conditions, we cannot rule out the possibility that it interacted selectively with other features in some way. Therefore, in Experiment 3, the impact of the feature "apex orientation" was controlled so that the blob hypothesis and the asymmetry hypothesis could be tested in a pure form.

\section{EXPERIMENT 3}

Experiment 3 was an exact replication of Experiment 2, with only one modification: all the angles in both the target and the background pointed in the same direction (see Figure 7). Detection of the target on the basis of differences in the orientation of the angle apexes was no longer possible. However, this modification to the stimulus is informative only if it does not simultaneously modify critical features relating to the blob and asymmetry hypotheses.

According to the blob hypothesis, this modification should not produce any change in the results. Rotating the angle by $180^{\circ}$ should not lead to any change in the perceived blobs. This is easy to understand by looking at Figure 2. If the angle in the ellipses is turned so that the apex takes the opposite direction, the form of the ellipses can be retained, and, thus, the orientation of their major axes can be retained as well. Also, according to the asymmetry hypothesis, detection performance should be unaffected by the stimulus modification. The larger or smaller angle widths remain.

Therefore, in Experiment 3, we anticipated the same pattern of results as that found in Experiment 2, because rotating the target angle in the same direction as the background angle changes neither the orientation of the blobs nor the size of the angle widths. We would not be surprised if total performance were to deteriorate, because a feature that discriminates between the target and the background - the direction of the angle apexes--has been removed.

\section{Method}

Subjects. Seven female and 3 male subjects were paid to participate in the experiment. All the subjects reported normal or fully corrected visual acuity.

Apparatus, Stimuli, and Procedure. These were the same as those in Experiments 1 and 2.

\section{Results}

As in Experiment 2, an ANOVA for repeated measures was computed with the variables of condition (same vs. different), position (1-35), and session $(2,3,4)$. There was a significant effect of condition $[F(1,9)=7.20, p=$ $.025]$, position $[F(34,306)=18.00, p<.001]$, and session $[F(2,18)=8.81, p=.002]$. There was also a significant interaction between condition and position $[F(34,306)=8.89, p<.001]$, but the other interactions did not attain significance (see Figure 8).

Orthogonal polynomial contrasts were calculated for the variable position. The results showed that the quadratic and the quartic effects of the variable position were different for both conditions [quadratic interaction condition/position, $F(1,9)=36.24, p<.001$; quartic, $F(1,9)=66.83, p<.001]$. This result indicates that the subtraction of the two conditions yields a value with a sign in the central area that is different from that in the two peripheral areas.

\section{Discussion}

The pattern of findings in Experiment 3 is completely analogous to that of Experiment 2. In the fovea, larger angles were detected better than were smaller angles.

a)
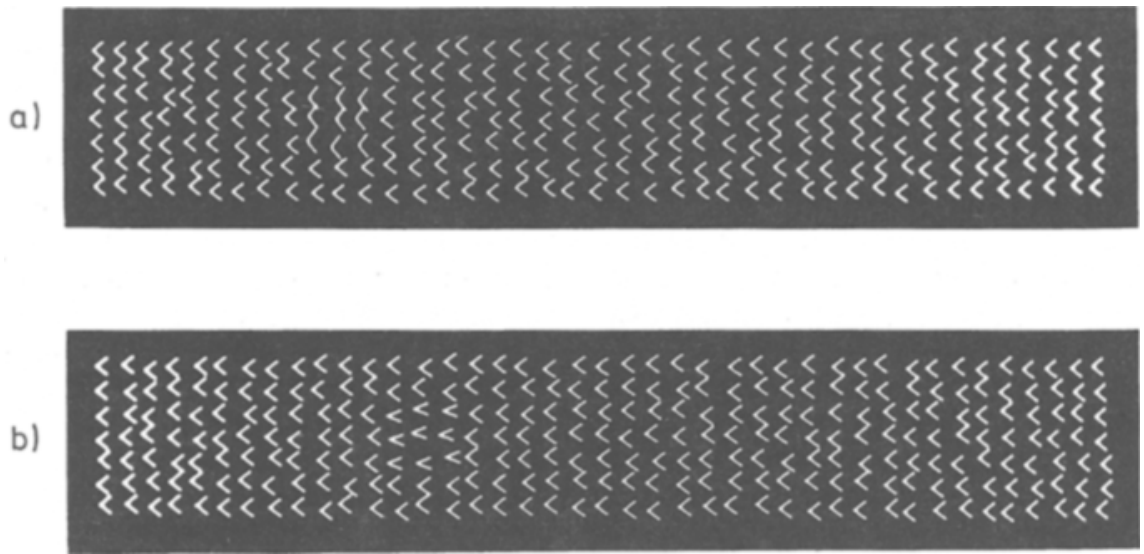

Figure 7. Stimulus patterns in Experiment 3. These are similar to those in Experiment 2, except all angle apexes point in the same direction. Angle width target/background: (a) same condition, $90^{\circ} / 70^{\circ}$; (b) different condition, $\mathbf{5 0} / 70^{\circ}$. 


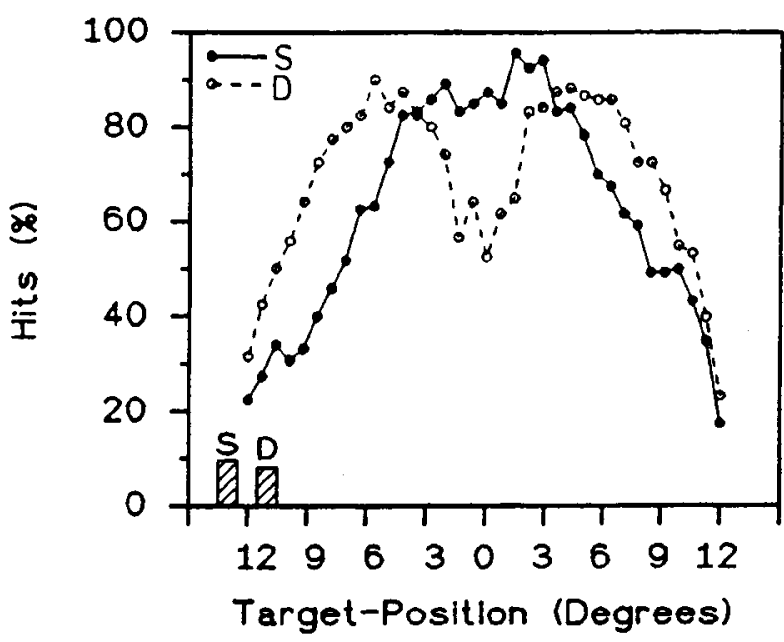

Figure 8. Percentage of hits as a function of retinal eccentricity (degrees) in the same condition (S) and the different condition (D) in Experiment 3. Blocks show false alarms.

Differences between the orientations of the blobs facilitated detection in the more peripheral areas. Thus, both the asymmetry and the blob hypotheses, as well as their relationship to retinal location, can be retained. The fact that in Experiment 3-unlike in Experiment 2-all the angle apexes in the target and background point in the same direction did not have a selective impact on segmentation performance in the fovea or periphery. Surprisingly, the stimulus manipulation did not lead to any major increase in task difficulty. It seems that the subjects did not exploit this discriminatory feature (differences in the orientations of the apexes) when detecting the target. However, this can only be concluded indirectly, as data from two different experiments are being compared.

\section{GENERAL DISCUSSION}

Let us return to our initial question. Starting with angle textures as used by, for example, Olson and Attneave (1970; see Figure 1), we have studied which stimulus features lead to their visual segmentation. On the basis of the ideas of Olson and Attneave (1970), Beck (1972), and Fox and Mayhew (1979), we have postulated that the individual texture elements are represented as blobs in the early visual system, and that the features of these virtual blobs determine segmentation performance (blob hypothesis). The present experiments have shown that features of blobs actually can be used to explain segmentation performance: If blobs in target and background exhibit different orientations of their major axes, segmentation performance is better than when no differences in orientation are present. However, the experiments have also shown that this relationship can be confirmed only for the more peripheral areas of the retina. Manipulations of the blob orientation are effective only beyond an eccentricity of approximately $3.5^{\circ}$. In the fovea, in contrast, segmentation performance is deter- mined by other features; differences in the size of angle widths seem to play a substantial role. Which general framework can be used to explain these findings? Do they have any implications for further research in the field of texture segmentation?

1. The representation of the stimulus within the visual system is not a one-to-one depiction of the physical stimulus; different features may be explicit in the stimulus and in the representation. The present study has confirmed that segmentation performance can be determined by features that are present only "indirectly" in the physical stimulus (orientation of the largest extent of angles). How can this be explained? The system-internal representation of the stimulus is not a one-to-one depiction of the physical stimulus. Features of the physical stimulus do not have to agree with features of the representation. In other words, other features are explicit in each case, depending on whether the physical stimulus or the stimulus representation is "observed." Features that are contained implicitly in the physical stimulus, such as the orientation of the largest extent of the angle, become more explicit in a specific stimulus representation, such as angle as blob. Mechanisms of the visual system use system-internal representations. It seems likely that the visual system gives preference to the further processing of the features that are explicit in these representations and that are, therefore, particularly accessible (see Kehrer \& Meinecke, 1994).

The conclusion for research in the field of texture segmentation is that, when searching for critical stimulus features, cases may arise in which such features cannot be taken directly from the physical stimulus. In such cases, how can we obtain information on the features that actually cause texture segmentation? Computer simulations may be useful here; they can be used to transform the physical stimulus in ways that are probably used by the visual system (e.g., Fogel \& Sagi, 1989; García Pérez, 1992; Griffiths \& Troscianko, 1992; Turner, 1986).

2 . The stimulus representation changes as a function of retinal eccentricity. The findings also show that different features influence texture segmentation as a function of retinal eccentricity. It is also possible to introduce an explanation for this: Anatomic and physiological properties of the visual system change as a function of retinal eccentricity (see De Valois \& De Valois, 1988). Some of these properties are, for example, the distribution of receptors across the retina (greater density in the fovea than in the periphery), the sensitivity for particular spatial frequencies (greater sensitivity for high frequencies in the fovea than in the periphery), or the socalled cortical magnification factor. These changes in the properties of the visual system lead to variations in system-internal stimulus representation as a function of retinal eccentricity. In other words, depending on the "retinal location" at which information processing is applied, different features are explicit because the stimulus representations differ.

As a result, cases may arise in research on texture segmentation in which it is not sufficient to search for the 
critical discriminating feature. It has to be considered that different features may be effective at different retinal eccentricities (see also Meinecke, 1989). However, information on these relationships is obtained only when segmentation performance is measured as a function of retinal eccentricity.

3. Peripheral representations do not necessarily lead to worse performance than foveal ones. A widely accepted assumption regarding the relation between retinal eccentricity and the performance of the visual system is that increasing eccentricity is accompanied by a drop in performance. This relationship has been confirmed repeatedly for a range of different phenomena (for references, see Yager \& Davis, 1987). However, our data show that this does not always have to be true, at least as far as texture segmentation is concerned. As Kehrer $(1987,1989)$ has already reported, some stimulus conditions reveal that optimal detection performance lies in more peripheral areas instead of in the fovea (similar results have been reported by Fiorentini, 1989, for visual search tasks). How does this finding fit into the framework presented here? As already mentioned, systeminternal representations of a stimulus differ as a function of retinal eccentricity, and, hence, different features are explicit in each case. Now, a case may arise in which features that are explicit in peripheral areas are precisely those that lead to texture segmentation. Then, the actual finding, as in some of the conditions in our experiments, should be a particularly good segmentation performance in these peripheral areas. Our data suggest that one of the explicit features in peripheral areas is the feature of "orientation," whereas the feature of "angle width" is one of the explicit features in the fovea.

In summary, our experiments show that the relationship between texture segmentation performance and retinal eccentricity can vary greatly. This can be explained adequately by considering that segmentation mechanisms in the early visual system address the stimulus representations and not the physical stimulus, that the stimulus representation varies as a function of eccentricity, and that this also leads to variation in the features that are explicit in each case. Further studies will be needed to evaluate which particular transformations in visual information processing bring about the findings reported here.

Kehrer and Meinecke (1993) have presented a first step in gaining some information about the influence of the varying receptive field size with eccentricity on the system-internal representation. The results of a computer simulation suggest that there is indeed an "optimal" receptive field size, in which segmentation performance is best, and that this receptive field has to be located at an eccentricity of about $5^{\circ}$ (according to the parameters proposed by Watson, 1983).

\section{REFERENCES}

BECK, J. (1966). Effect of orientation and of shape similarity on perceptual grouping. Perception \& Psychophysics, 1, 300-302.
BECK, J. (1972). Similarity grouping and peripheral discriminability under uncertainty. American Journal of Psychology, 85, 1-20.

BECK, J. (1982). Textural segmentation. In J. Beck (Ed.), Organization and representation in perception (pp. 285-317). Hillsdale, NJ: Erlbaum.

Beck, J., Graham, N., \& SutTer, A. (1991). Lightness differences and the perceived segregation of regions and populations. Perception \& Psychophysics, 49, 257-269.

BERGEN, J. R. (1991). Theories of visual texture perception. In D. Regan (Ed.), Vision and visual dysfunction (Vol. 10, pp. 114134). New York: Macmillan.

BERGEN, J. R., \& ADELSON, E. H. (1988). Early vision and texture perception. Nature, 333, 363-364.

BERGEN, J. R., \& JULESZ, B. (1983). Rapid discrimination of visual patterns. IEEE Transactions on Systems, Man, \& Cybernetics, 13, 857-863.

Caelli, T. (1985). Three processing characteristics of visual texture segmentation. Spatial Vision, 2, 19-30.

Caelli, T., \& Julesz, B. (1979). Psychophysical evidence for global feature processing in visual texture discrimination. Journal of the Optical Society of America, 69, 675-678.

CARTER, R. C. (1982). Visual search with color. Journal of Experimental Psychology: Human Perception \& Performance, 8, 127-136.

Cavanagh, P., Arguin, M., \& Treisman, A. (1990). Effect of surface medium on visual search for orientation and size features. Journal of Experimental Psychology: Human Perception \& Performance, 16, 479-491.

Deubel, H., Findlay, J., Jacobs, A. M., \& Brogan, D. (1988). Saccadic eye movements to targets defined by structure differences. In G. Luer, U. Lass, \& J. Shallo-Hoffmann (Eds.), Eye movement research: Physiological and psychological aspects (pp. 107-145). Toronto: Hogrefe.

DeUREL, H., \& FranK, H. (1991). The latency of saccadic eye movements to texture-defined stimuli. In R. Schmid \& D. Zambarbieri (Eds.), Oculomotor control and cognitive processes: Normal and pathological aspects (pp. 369-384). Amsterdam: Elsevier, North-Holland.

De ValoIs, R. L., \& De ValoIs, K. K. (1988). Spatial vision. New York: Oxford University Press.

ENGEL, F. L. (1974). Visual conspicuity and selective background interference in eccentric vision. Vision Research, 14, 459-471.

FARMER, E. W., \& TAYLOR, R. M. (1980). Visual search through color displays: Effects of target-background similarity and background uniformity. Perception \& Psychophysics, 27, 267-272.

FIORENTINI, A. (1989). Differences between fovea and parafovea in visual search processes. Vision Research, 29, 1153-1164.

FoGEL, I., \& SAGI, D. (1989). Gabor filters as texture discriminator. Biological Cybernetics, 61, 103-113.

Fox, J., \& MaYhew, J. E. W. (1979). Texture discrimination and the analysis of proximity. Perception, 8, 75-91.

García PÉREz, M. A. (1992). The perceived image: Efficient modelling of visual inhomogeneity. Spatial Vision, 6, 89-99.

Graham, N. (1989). Low-level visual processes and texture segregation. Physica Scripta, 39, 147-152.

Griffiths, E., \& Troscianko, T. (1992). Can human texture discrimination be mimicked by a computer model using local Fourier analysis? Spatial Vision, 6, 149-157.

Gurnsey, R., \& BRowSE, R. A. (1987). Micropattern properties and presentation conditions influencing visual texture discrimination. Perception \& Psychophysics, 41, 239-252.

GURNSEY, R., \& BROWSE, R. A. (1989). Asymmetries in visual texture discrimination. Spatial Vision, 4, 31-44.

KeHRer, L. (1987). Perceptual segregation and retinal position. Spatial Vision, 2, 247-261

KeHRER, L. (1989). Central performance drop on perceptual segregation tasks. Spatial Vision, 4, 45-62.

Kehrer, L., \& Meinecke, C. (1993). Orientation differences in texture segmentation: Psychophysical data and a spatial filter model. Perception, 22(Suppl.), 71-72.

Kehrer, L., \& Meinecke, C. (1994). Perzeptive Organisation visueller Muster: Die Segmentierung von Texturen. In W. Prinz \& B. Bridgeman (Eds.), Enzyklopädie der Psychologie: Band C/II/I. Wahrnehmung. Göttingen: Hogrefe. (English translation in preparation.) 
MARR, D. (1976). Early processing of visual information. Philosophical Transactions of the Royal Society of London: Series B, 275, 483-524.

McIlhagga, W., Hine, T., Cole, G. R., \& SNydeR, A. W. (1990). TeXture segregation with luminance and chromatic contrast. Vision Research, 30, 489-495.

MEINECKE, C. (1989). Retinal eccentricity and the detection of targets. Psychological Research, 51, 107-116.

NothDURFT, H. C. (1985a). Orientation sensitivity and texture segmentation in patterns with different line orientation. Vision Research, 25, 551-560.

NoTHDURFT, H. C. (1985b). Sensitivity for structure gradient in texture discrimination tasks. Vision Research, 25, 1957-1968.

Nothdurf, H. C. (1990). Texton segregation by associated differences in global and local luminance distribution. Proceedings of the Royal Society of London: Series B, 239, 295-320.

Nothdurf, H. C. (1991). Texture segmentation and pop-out from orientation contrast. Vision Research, 31, 1073-1078.

Olson, R. K., \& ATtNeAve, F. (1970). What variables produce similarity grouping? American Journal of Psychology, 83, 1-21.
SACHS, L. (1978). Angewandte Statistik. Berlin: Springer-Verlag.

SAGI, D., \& JULESZ, B. (1987). Short-range limitation on detection of feature differences. Spatial Vision, 2, 39-49.

Treisman, A. M., \& Gelade, G. (1980). A feature-integration theory of attention. Cognitive Psychology, 12, 97-136.

TURNER, M. R. (1986). Texture discrimination by Gabor functions. Biological Cybernetics, 55, 71-82.

WatSon, A. B. (1983). Detection and recognition of simple spatial forms. In O. J. Braddick \& A. C. Sleigh (Eds.), Physical and biological processing of images (pp. 100-114). New York: Springer-Verlag.

YAGER, D., \& DAVIS, E. T. (Eds.) (1987). Variations of visual functions across the visual field. Journal of the Optical Society of America A (Special Feature), 4, 1481-1703.

(Manuscript received February 10, 1993; revision accepted for publication February 22, 1994.) 